\section{From spectrum to molecule}

\section{William Rhodes}

Theory and Methods of Calculation of Molecular Spectra. By L. A. Gribov and W. J. Orville-Thomas. Wiley: 1988. Pp. 636. \$178, £86.

ONE of the central themes of molecular spectroscopy is the interplay between molecular structure and spectral structure, and how this interplay is governed by the relative motions of the electrons and nuclei in the molecule. Although the theoretical basis is well understood in terms of the Born-Oppenheimer scheme and its ramifications, it is suprising that there are few books which treat the subject in sufficient detail to provide a proper grounding for students entering the field. Any serious attempt to remedy the situation should therefore be appreciated. Gribov and Orville-Thomas's book is presumably such an attempt; it is aimed not only at the beginner, but also at those who wish to understand rather sophisticated methods of relating molecular and spectral structure.

The first five chapters give an elementary, but wide-ranging, treatment of the adiabatic (Born-Oppenheimer) approximation, the Franck-Condon principle, vibronic coupling, molecular-electronic structure, symmetry and related subjects. The following seven offer a detailed formulation of such specific topics as kinematic coefficients, the harmonic approximation, force constants and infrared spectra. Finally, there are three chapters which contain a symbolic logic approach to spectroscopy and methods of calculating electronic and Raman spectra. Throughout, there is an emphasis on developing spectroscopic quantities in terms of elementary molecular entities, such as atomic orbitals and internuclear geometric parameters. This makes for a lot of detailed algebra.

To be sure, Gribov and Orville-Thomas bring their own flair and flavour to bear on the subject. They are clearly experts in this field and have developed a perspective and methodology in a way that few (if any) others could do. Nevertheless, the book is not without shortcomings. In the preface, the authors say that their purpose is to show "how computers can be used to supplement, and in some cases to replace, experimental spectroscopic studies

Yet, except for Chapter 13, which deals with symbolic logic, the book has little to do directly with computing. It does not even bring the reader to the brink of computing, although the stress throughout is on algebraic formulation of quantities and parameters which could easily be subjected to computational algorithms.
Furthermore, the authors do not seem to have a definite audience in mind. Many chapters blend introductory explanations and sophisticated, abstract formulations in a manner which seems to be pedagogically inconsistent. For example, in Section 3.1 great care is taken to explain the meaning of hermitian operators, yet elsewhere in the book many other operations and concepts that are less widely known are used with little explanation. The simplest concepts of symmetry are introduced pictorially in Section 5.1, yet this is followed by a sophisticated technique involving circulants rather than the usual group-theory approach. The point is that anyone who needs the pictorial introduction is quite likely to be bewildered by the algebra that follows.

Perhaps the most serious criticism applies to the first three chapters, which lay the theoretical foundations. Here, the authors seem more intent on justifying their practical approach to molecular spectroscopy, in a manner that is oblique and discursive, and even philosophical at times, rather than on making a clear exposition of the basics for the beginner. Considerable emphasis is placed on what is called the "inverse spectral problem", a method of adapting theoretical models to experimental spectra, but one which will probably remain nebulous to the reader until Chapter 13, where it is applied. The early chapters also contain a number of pedagogical errors and poorly drawn figures. These include incorrect statements about the relative value of electronic and vibrational energies (p. 22), impossible curves for vibrational wavefunctions (Fig. 2.7 ), and an unnecessary obfuscation of the mirror symmetry law of absorption and emission spectra depicted in Fig. 2.8.

These weaknesses in the underlying fundamentals, together with the fact that calculational methods per se are not dealt with, suggest that perhaps a better title would have been Theoretical Methods of Molecular Spectroscopy. But as a book on the ways of relating spectral parameters to molecular parameters, it is quite strong and should be a valuable resource to anyone who wishes to go beyond the basics of the subject.

William Rhodes is a Professor in the Institute of Molecular Biophysics and Department of Chemistry, Florida State University, Tallahassee, Florida 32306, USA.

\section{World of decay}

\section{Adrian Bath}

Isotopes In the Earth Sciences. By Robert Bowen. Elsevier Applied Science: 1989. Pp. 647. £80, \$144.

STUDIEs of natural isotope systems have had a vast influence on the Earth sciences. They are one of the most effective ways by which to quantify geosphere parameters - time, temperature, source identification and mixing - and have been a driving force for technical innovation in the sophisticated and expensive business of isotopic measurement. Robert Bowen addresses his book to a broad range of Earth and environmental scientists as a comprehensive guide through the scientific and technical complexities of the subject.

Since its early days, isotope geoscience has been dominated by its geochronological applications, in much the same way that the immediate relevance of carbon-14 dating has appealed to archaeologists. This historical dominance is reflected by half of the book dwelling on geological dating and its interpretative problems. To many it must seem that isotope studies create or define as many problems as they solve; they illustrate how complex and intractable Earth processes are.

The second half takes a lateral view of the roles of isotopes in our understanding of the spectrum of processes in geospherehydrosphere-biosphere systems. This is the most exciting aspect of the subject, but treatment here of the assorted topics varies between excessive detail and undue superficiality, and never finds a satisfactory balance or unifying approach. The final chapter covers something entirely different - the historical and political background to nuclear power and radioactive waste disposal, presumably in recognition of their perceived link with all isotopes.

Bowen's book is essentially an encyclopaedic and uncritical catalogue of developments in isotope studies up to the present. Disappointingly little space is devoted to looking ahead. New research needs to be directed towards predictive Earth studies and towards understanding the difficult but challenging interfaces between the geosphere, hydrosphere and biosphere. The Earth scientist is unique in being concerned with processes over widely ranging timescales in the past. Prediction of the future is a logical but difficult progression, and our challenge is to seek out further ways in which isotopes will improve the scientific basis of such prediction. It hardly needs adding that, in an area of research which demands ever greater investment of resources, there is a need to educate an audience beyond the already-convinced Earth scientist.

Adrian Bath is in the Fluid Processes Research Group, British Geological Survey, Keyworth Nottinghamshire NG12 5GG, UK.

- The Uranium Institute, London, has published the proceedings of its most recent symposium under the title Uranium and Nuclear Energy: 1988. Price is $£ 52, \$ 100$. 